\title{
Late relapse in stage I of nonseminomatous germ cell testicular cancer on surveillance
}

\author{
Ondrusova $\mathrm{M}^{1,2}$, Suchansky $\mathrm{M}^{2}$, Psota $\mathrm{M}^{2}$, Zeleny $\mathrm{T}^{3}$, Ondrus $\mathrm{D}^{4}$ \\ St. Elisabeth University of Health and Social Sciences, Bratislava, Slovakia. dalibor.ondrus@ousa.sk
}

\begin{abstract}
OBJECTIVE: Primary aim was to assess relapse-free survival (RFS) in patients with clinical stage I (CS I) of non-seminomatous germ cell testicular tumors (NSGCTT) undergoing surveillance after orchiectomy. The secondary aim was to examine differences in risk factors in patients with early relapse (ER $<2$ years), late relapse (LR > 2 years) and very late relapse (VLR > 5 years).

METHODS: Cross-sectional study analyzed 25-year single-center experiences with 198 CS I NSGCTT patients according the time to relapse.

RESULTS: RFS was 160/198 (80.8\%). Relapse occurred in 38 (19.2\%) patients after a median follow-up of 7.57 months, 33 (86.8 \%) patients had ER after a median follow-up of 7.03 months and 5 patients had LR (13.2\%) after a median follow-up of 26.28 months. One patient (2.63 \%) had VLR after follow-up > 5 years (7.17 years). Three relapsed patients died with metastatic disease after a mean follow-up of 5.1 years from the date of diagnosis. Another three patients died without cancer after a mean follow-up of two years. OS was 192/198 (97\%). CONCLUSION: Diagnosis and treatment of late relapsing NSGCTT patients should be performed in experienced centers only. Occurrence of LR is the reason for long-term monitoring of NSGCTT survivors (Tab. 1, Fig. 1, Ref. 14). Text in PDF www.elis.sk.

KEY WORDS: testicular cancer, active surveillance, clinical stage, late relapse.
\end{abstract}

\section{Introduction}

Comprehensive data on late relapse (LR) and very LR (VLR) in patients with clinical stage I (CS-I) of testicular cancer followed on surveillance are sparse (1). Most relapses of germ cell tumors occur within 2 years of initial treatment. In $2-4 \%$ of patients, relapse may occur later (2). Most investigators define LR as recurrence at least 2 years after completion of successful primary treatment and differentiate between those occurring before and after 5 years (VLR) $(3,4,5,6)$. Primary aim of the present study was to assess the incidence and clinical outcome of chemotherapy-naïve patients with LR in a cohort of CS-I patients undergoing surveillance. The secondary aim was to examine differences in risk factors in patients with early relapse (ER), LR and VLR.

\section{Patients and methods}

The cross-sectional study was carried out from 1/1992 to $8 / 2017$ in a single center particularly dedicated to the diagnosis

${ }^{1}$ St. Elisabeth University of Health and Social Sciences, Bratislava, Slovakia, ${ }^{2}$ Pharm-In, Bratislava, Slovakia, ${ }^{3}$ Institute of Hygiene and Epidemiology, 1st Faculty of Medicine, Charles University and General University Hospital in Prague, Prague, Czech Republic, and ${ }^{4} 1$ st Department of Oncology, Comenius University Faculty of Medicine, Bratislava, Slovakia

Address for correspondence: D. Ondrus, MD, DSc, 1st Department of Oncology, Comenius University Faculty of Medicine, St. Elisabeth Cancer Institute, Heydukova 10, SK-812 50 Bratislava, Slovakia. and treatment of testicular cancer. This study included all newly diagnosed patients with CS-I nonseminomatous germ cell testicular tumors (NSGCTT) $(\mathrm{n}=198$ patients (followed up for at least 10 years after orchiectomy) who were without vascular invasion (pT1) in the primary tumor. Patients with choriocarcinoma components were not included in the study.

Patients were assigned to the particular clinical stages on the basis of physical examination, CT examination of the chest, abdomen and pelvis, postorchiectomy levels of serum tumor markers (STM) - alpha fetoprotein (AFP), and human chorionic gonadotropin $(\beta-h C G)$. The criteria for inclusion into CS-I were normal values of all these examinations. Informed consent was obtained from all patients (according to inclusion criteria mentioned above). As a result, none of the patients were excluded.

All performed diagnostic and therapeutic approaches were in accordance with the latest guideline recommendations for patients with testicular cancer $(7,8,9)$. All examinations and outcomes were prospectively and consecutively recorded.

Eligible patients were managed by risk-adapted therapeutic approach (policy of surveillance), which consisted of a regular follow-up after orchiectomy with STM (AFP, $\beta$-hCG) measurement and abdominal CT scans performed every 3 months in the first year, twice yearly for the next 2-5 years, and annually thereafter. Patients with retroperitoneal disease on CT scans underwent consequently chest examinations.

Time to relapse was defined as the time from date of orchiectomy to date of relapse diagnosis. 
Patients were divided into two groups, namely those with early relapses (ER) ( $\geq 3$ months and $<2$ years after orchiectomy) and those with late relapses ( $\geq 2$ years after orchiectomy). A shorter term of three months for ER was chosen to exclude patients with synchronous metastatic disease. Patients relapsing during followup were treated with platinum-based combination chemotherapy - BEP regimen (bleomycin $30 \mathrm{U}$ IV on days 1 , 8, and 15 plus etoposide $100 \mathrm{mg} / \mathrm{m}^{2} \mathrm{IV}$ on days $1-5$ plus cisplatin $20 \mathrm{mg} / \mathrm{m}^{2} \mathrm{IV}$ on days $1-5$; every 21 days).

\section{Statistical analysis}

The demographic characteristics of all patients were analyzed by descriptive statistics. Potential differences in patient characteristics between ER and LR (VLR) were assessed by $\chi^{2}$ test or Fisher exact test for categorical variables and Mann-Whitney $U$ test for continuous variables. We compared characteristics as follows: age, STM (AFP and $\beta$-hCG) elevation at the time of relapse as well as presence of embryonal carcinoma, yolk sac tumor and teratoma at the time of diagnosis. Data analysis was carried out using statistical software R version 3.0. All statistical tests were two-sided and statistical significance was set at $\mathrm{p}<0.05$. The Kaplan-Meier method was used to estimate overall survival and relapse-free survival.

\section{Results}

The median age of 198 CS-I NSGCTT patients at the time of diagnosis was 27.9 years (interquartile range [IQR] 23.6 and 33.9; range 15.1-57.5) (Tab. 1).

Median follow-up for 198 patients was 17.03 years (IQR was 12.8-21.4; range 2.7 to 24.5 ).

Relapse free survival was 160/198 (80.8\%). Relapse occurred in $38(19.2 \%)$ patients after a median follow-up of 7.57 months (IQR 5.31-14.95; range 3.45 to 86.0) (Fig. 1).

Thirty-three $(86.8 \%)$ patients had had ER $(<2$ years $)$ after a median follow-up of 7.03 months (IQR 5.16-11.76).

Five patients had LR (13.2\%) ( $\geq 2$ years) after a median follow-up of 26.28 months (IQR 24.02-31.38).

One of all relapsed patients $(2.63 \%)$ had relapse beyond 5 years ( 7.17 years) after follow-up (VLR).

\section{Tab. 1. Patients characteristics.}

\begin{tabular}{lc}
\hline Patients characteristics & Summa \\
\hline number of patients & 198 \\
median age at the time of diagnosis (years) & 27.9 \\
range & $15.1-57.5$ \\
\hline Histology of the primary tumor & $155(78.3)$ \\
$\quad$ presence of embryonal carcinoma, n (\%) & $155(78.3)$ \\
$\quad$ presence of teratoma, $\mathrm{n}(\%)$ & $44(22.2)$ \\
$\quad$ presence of Yolk sac tumor, n (\%) & $19(50.0)$ \\
\hline Serum tumor markers at the time of relapse & $17(44.7)$ \\
$\quad$ elevated AFP, $\mathrm{n}(\%)$ & 7.6 \\
$\quad$ elevated $\beta$ hCG, $\mathrm{n}(\%)$ & $3.45-86.0$ \\
$\quad$ median time to relapse (months) & $38(19.2)$ \\
$\quad$ range & $160(81)$ \\
relapse rate, $\mathrm{n}(\%)$ & $192(97)$ \\
$\quad$ relapse free survival, $\mathrm{n}(\%)$ & \\
$\quad$ overall survival, $\mathrm{n}(\%)$ &
\end{tabular}

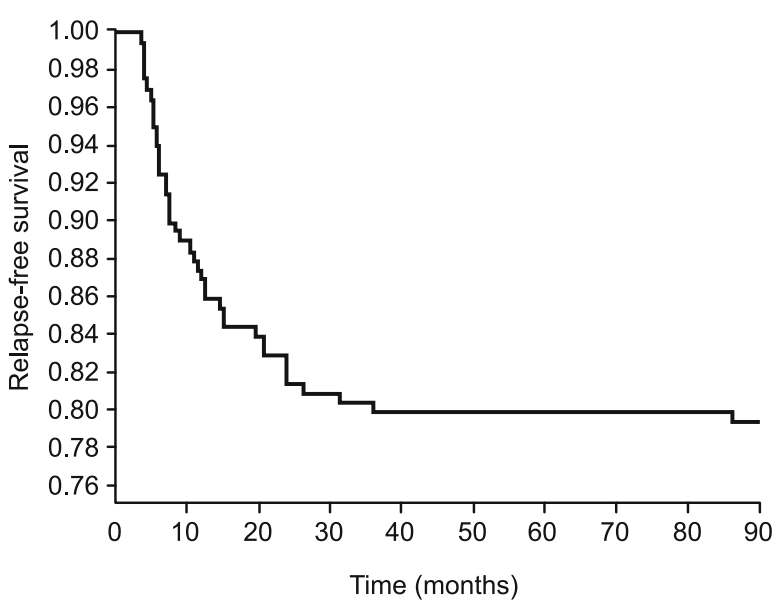

Fig. 1. Relapse-free survival for CS-I NSGCTT patients.

Three relapsed patients died after a mean follow-up of 5.1 years (range, 2.7 to 8.5 ) from the date of diagnosis with metastatic disease progression. Another three patients died without oncological disease after a mean follow-up of 2.0 years (range, 0.95 to 3.0) from the date of diagnosis.

The overall survival (OS) was 192/198 (97\%). Comparing OS of patients with relapse $(35 / 38,92.1 \%)$ and patients without relapse (157/160 [98.1\%]), we found no significant differences. Comparing OS of patients with ER (31/33 [93.9\%]) and patients with LR (4/5 [80\%]), we found no significant differences.

Relapses of $28 / 38$ (73.7\%) patients were located only in the retroperitoneum (stage IIA -6 patients, stage IIB - 21 patients, stage IIC - one patient) while 10/38 (26.3\%) patients were observed to develop lung metastases.

Elevation of AFP at the time of relapse was observed in 16 patients with ER (48.5\%) and in three patients with LR (60\%). The difference was not statistically significant.

At the time of relapse more patients with $\operatorname{ER}(n=15,45.5 \%)$ than those with LR (VLR) ( $\mathrm{n}=$ two, $40 \%$ ) had elevated levels of $\beta$-hCG, but the difference was not statistically significant.

All relapses were detected by CT scan at routine examination.

Embryonal carcinoma components in the primary tumor was observed in 125/160 (78.1 patients without relapse and in 30/38 (78.9\%) patients with relapse. There were no significant differences in primary histology between relapsed and non-relapsed patients.

The presence of embryonal carcinoma components in the primary tumor was observed in 27/33 (81.8\%) patients with ER and in $3 / 5(60 \%)$ patients with LR. There were no significant differences in primary histology between ER and LR patients.

The presence of teratoma components in the primary tumor was observed in 124/160 (77.5\%) patients without relapse and in $31 / 38(81.6 \%)$ patients with relapse. There were no significant differences in primary histology between relapsed and nonrelapsed patients.

Comparing ER with LR, the presence of teratoma components in the primary tumor was observed in $26 / 33(78.8 \%$ ) patients with 
ER and in all 5 (100\%) patients with LR. There were no significant differences in primary histology between ER group and LR group.

There were significant differences only in presence of yolk sac tumor in the primary tumor between group of patients without relapse and group of patients with relapse, namely 30/160 (18.7 $\%)$ patients and 14/38 (36.8\%) patients, respectively $(\mathrm{p}<0.05)$.

Comparing ER and LR patients, yolk sac tumor in the primary tumor was observed in 12/33 (36.4\%) patients with ER and in 2/5 (40\%) patients with LR. There were no significant differences in primary histology between ER group and LR group.

Complete response (CR) after BEP chemotherapy alone was obtained in 19/38 (50 \%) patients (four in stage IIA, 11 in stage IIB, four in stage III). Postchemotherapy retroperitoneal lymph node dissection (RPLND) was performed in 11 patients (28.9\%; 8 patients with mature teratoma, two patients with necrosis, one patients with viable tumor). Primary RPLND was done in two patients (one mature teratoma and one viable tumor). Four patients underwent pulmonary resection (three patients with fibrosis and one with viable tumor). Patients with viable tumor were treated with additional chemotherapy. On the last follow-up 35/38 (92.1\%) patients were considered tumor-free after treatment of the relapse.

\section{Discussion}

Late relapse of germ cell tumor is a well-recognized entity associated with poor survival (10). Fedyanin et al (11) observed a significantly worse outcome in patients with LR than in patients with ER, namely the complete response rate after induction chemotherapy was $20.7 \%$ versus $42.1 \%$. Very late relapse (VLR) after 5 years is a rare event occurring in approximately $0.5 \%$ of patients according to a population-based analysis (6) Therefore, the aim of follow up beyond 5 years shifts to the detection of late side effects of treatment. For CS-I NSGCTT on active surveillance, the relapse rate after 5 years is $0.3 \%$ according to a large recent analysis (1). These results were confirmed in another cohort (12). In view of these data it is debatable, if regular followup of all testicular cancer patients beyond 5 years is a good use of medical resources. Most patients with VLR are diagnosed due to symptoms, however in up to $50 \%$, elevated STM can be found in both seminomas and NSGCTT $(1,6)$. Patient education about relapse symptoms and physician awareness is a very important part of survivorship management. The early use of imaging and STM in case of suspicion of relapse is encouraged. Daugaard et al (13) observed VLR in $1.6 \%$ of patients. They noted, that the frequency of relapses after 5 years is only sporadically described because most studies have much shorter observation time. In our present study, we observed VLR in $2.63 \%$ of relapsing patients. Optimal treatment for chemotherapy-naïve patients with late-relapsing NSGCTT should be based on a representative biopsy and included radical surgery (using interdisciplinary team of urologists, surgeons, oncologists, and radiologists and possibly also vascular, thoracic, orthopedic specialists and neurosurgeons) and subsequent chemotherapy in most cases. Diagnosis and treatment of late relapsing NSGCTT patients is challenging and should be performed in experienced (high-volume) centers only (14).
According to our experience, the occurrence of late relapses is the reason for long-term (life-long) monitoring of testicular cancer survivors. It makes sense for early diagnosis of secondary malignancies.

\section{References}

1. Mortensen MS, Lauritsen J, Kier MG, Bandak M, Appelt AL, Agerbæk M, Holm NV et al. Late relapses in Stage I testicular cancer patients on surveillance. Eur Urol 2016; 70 (2): 365-371.

2. Ehrlich Y, Rosenbaum E, Baniel J. Late relapse of testis cancer. Curr Urol Rep 2013; 14 (5): 518-524.

3. Baniel J, Foster RS, Gonin R, Messemer JE, Donohue JP, Einhorn LH. Late relapse of testicular cancer. J Clin Oncol 1995; 13(5): 1170-1176.

4. Gerl A, Clemm C, Schmeller N, Hentrich M, Lamerz R, Wilmanns W. Late relapse of germ cell tumors after cisplatin-based chemotherapy. Ann Oncol 1997; 8 (1): 41-47.

5. Dieckmann KP, Albers P, Classen J, De Wit M, Pichlmeier U, Rick $\mathbf{O}$, Müllerleile $\mathbf{U}$ et al. Late relapse of testicular germ cell neoplasms: a descriptive analysis of 122 cases. J Urol 2005; 173 (3): 824-829.

6. Oldenburg J, Alfsen GC, Wæhre H, Fosså SD. Late recurrences of germ cell malignancies: a population-based experience over three decades. Br J Cancer 2006; 94 (6): 820-827.

7. Beyer J, Albers P, Altena R, Aparicio J, Bokemeyer C, Busch J, Cathomas $\mathbf{R}$ et al. Mainaining success, reducing treatment burden, focusing on survivorship: highlight from the third European consensus conference on diagnosis and treatment of germ-cell cancer. Ann Oncol 2013; 24 (4): 878-888.

8. Oldenburg J, Fosså SD, Nuver J, Heidenreich A, Schmoll HJ, Bokemeyer C, Horwich A et al. Testicular seminoma and non-seminoma: ESMO Clinical Practice Guidelines for diagnosis, treatment and followup. Ann Oncol 2013; Suppl 6: vi125-vi132.

9. Albers P, Albrecht W, Algaba F, Bokemeyer C, Cohn-Cedermark G, Horwich A, Laguna MP et al. Guidelines on testicular cancer: 2015 Update. Eur Urol 2015; 68 (6): 1054-1068.

10. Sharp DS, Carver BS, Eggener SE, Kondagunta GV, Motzer RJ, Bosl GJ, Sheinfeld J et al. Clinical outcome and predictions of survival in late relapse of germ cell tumor. J Clin Oncol 2008; 26 (34): 5524-5529

11. Fedyanin M, Tryakin A, Kanagavel D, Bulanov A, Burova A, Figurin K, Fainshtein I et al. Late relapses ( $>2$ years) in patients with stage I testicular germ cell tumors: Predictive factors for survival. Urol Oncol 2013; 31 (4): 499-504.

12. Kollmannsberger C, Tandstad, T, Bedard PL, Cohn-Cedermark G, Chung PW, Jewett MA, Powles T et al. Patterns of relapse in patients with clinical stage I testicular cancer managed with active surveillance. J Clin Oncol 2015; 33 (1): 51-57.

13. Daugaard G, Gundgaard MG, Mortensen MS, Agerbæk M, Holm NV, Rørth M, von der Maase $H$ et al. Surveillance for stage I nonseminoma testicular cancer: outcomes and long-term follow-up in a populationbased cohort. J Clin Oncol 2014; 32 (34): 3817-3823.

14. Oldenburg J, Martin JM, Fosså SD. Late relapses of germ cell malignacies: incidence, management and prognosis. J Clin Oncol 2006; 24 (35): 5503-5511.

Received July 26, 2017. Accepted August 16, 2017. 